\title{
Approximation by Durrmeyer-type operators
}

\author{
by Vijay Gupta and G. S. SRivastava (Roorkee)
}

\begin{abstract}
We define a new kind of Durrmeyer-type summation-integral operators and study a global direct theorem for these operators in terms of the Ditzian-Totik modulus of smoothness.
\end{abstract}

1. Durrmeyer [4] introduced modified Bernstein polynomials to approximate Lebesgue integrable functions on $[0,1]$, later motivated by the integral modification of Bernstein polynomials by Durrmeyer; Sahai and Prasad [9] and Mazhar and Totik [8] introduced modified Lupas operators and modified Szász operators respectively to approximate Lebesgue integrable functions on $[0, \infty)$. A lot of work has been done on these three operators (see e.g. [1], [2], [7]-[10] etc.). In a recent paper Heilmann [6] has studied the generalized operators which include all the three operators. We now give another generalization of these operators as

$$
M_{n}(f, x)=\sum_{k=0}^{\infty} p_{n, k}(x) \int_{0}^{\infty} b_{n, k}(t) f(t) d t,
$$

where

$$
p_{n, k}(x)=(-1)^{k} \frac{x^{k}}{k !} \phi_{n}^{(k)}(x), \quad b_{n, k}(t)=(-1)^{k+1} \frac{t^{k}}{k !} \phi_{n}^{(k+1)}(t)
$$

and

(i) $\quad \phi_{n}(x)=(1+c x)^{-n / c} \quad$ for the interval $[0, \infty)$ with $c>0$,

(ii) $\quad \phi_{n}(x)=e^{-n x} \quad$ for the interval $[0, \infty)$ with $c=0$,

(iii) $\quad \phi_{n}(x)=(1-x)^{n} \quad$ for the interval $[0,1]$ with $c=-1$.

1991 Mathematics Subject Classification: 41A30, 41A36.

Key words and phrases: modulus of smoothness, global direct theorem, differential and integral operators.

Research of the first author supported by Council of Scientific and Industrial Research, India. 
The cases (i), (ii) and (iii) mentioned above give modified Baskakov type operators, modified Szász-type operators and modified Bernstein type polynomials respectively. The case (i) for $c=1$ was recently introduced by one of the authors (see e.g. [5]).

By $\mathfrak{L}_{1}^{r}[0, \infty)$ we denote the class of functions $g$ given by $\mathfrak{L}_{1}^{r}[0, \infty)=\{g$ : $g^{(r)} \in L_{1}[0, a]$ for every $a \in(0, \infty)$ and $\left|g^{(r)}(t)\right| \leq M(1+t)^{m}, M$ and $m$ are constants depending on $g\}$.

We remark that $L_{p}^{r}[0, \infty)$ is not contained in $\mathfrak{L}_{1}^{r}[0, \infty)$ and $L_{1}^{0}[0, \infty)=$ $L_{1}[0, \infty)$.

Following [3], the modulus of smoothness is given by

$$
\omega_{\phi}^{2}(f, t)_{p}=\sup _{0<h \leq t}\left\|\Delta_{h \phi}^{2} f\right\|_{p}, \quad \phi(x)=\sqrt{x(1+c x)},
$$

where

$$
\Delta_{h}^{\phi} f(x)= \begin{cases}f(x-h)-2 f(x)+f(x+h) & \text { if }[x-h, x+h] \subseteq[0, \infty), \\ 0 & \text { otherwise. }\end{cases}
$$

This modulus of smoothness is equivalent to the modified $K$-functional (see e.g. [3]) given by

$$
\bar{K}_{\phi}^{2}\left(f, t^{2}\right)=\inf \left\{\|f-g\|_{p}+t^{2}\left\|\phi^{2} g^{\prime \prime}\right\|_{p}+t^{4}\left\|g^{\prime \prime}\right\|: g \in \bar{W}_{p}^{2}(\phi,[0, \infty))\right\},
$$

where

$$
\bar{W}_{p}^{2}(\phi,[0, \infty))=\left\{g \in L_{p}[0, \infty): g^{\prime} \in \mathrm{AC}_{\mathrm{loc}}[0, \infty), \phi^{2} g^{\prime \prime} \in L_{p}[0, \infty)\right\} .
$$

In the present paper, we give a global direct theorem for the operators (1.1) in terms of the Ditzian-Totik modulus of second order. Throughout the paper, we denote by $C$ positive constants not necessarily the same at each occurrence.

2. In this section, we mention certain properties and results for the operators (1.1), which are necessary for the proof of the main result.

For the cases (i) and (ii), we have

$$
\begin{aligned}
& \sum_{k=0}^{\infty} p_{n, k}(x)=1, \quad \sum_{k=0}^{\infty} b_{n, k}(t)=n \\
& \int_{0}^{\infty} p_{n, k}(x) d x=\frac{1}{n-c} \text { and } \quad \int_{0}^{\infty} b_{n, k}(t) d t=1,
\end{aligned}
$$

and for the case (iii) summation is from 0 to $n$ and integration from 0 to 1 .

For $\phi(x)=\sqrt{x(1+c x)}$, we have

$$
\begin{aligned}
\phi^{2}(x) p_{n, k}^{(1)}(x) & =[k-n x] p_{n, k}(x), \\
\phi^{2}(t) b_{n, k}^{(1)}(t) & =[k-(n+c) t] b_{n, k}(t) .
\end{aligned}
$$


Lemma 1. For $m, r \in \mathbb{N}^{0}$ (the set of non-negative integers), if we define

$$
V_{r, n, m}(x)=\sum_{k=0}^{\infty} p_{n+c r, k}(x) \int_{0}^{\infty} b_{n-c r, k+r}(t)(t-x)^{m} d t,
$$

then

$$
V_{r, n, 0}(x)=1, \quad V_{r, n, 1}(x)=\frac{(1+r)+c x(1+2 r)}{n-c(r+1)}
$$

and

$$
\begin{aligned}
& V_{r, n, 2}(x) \\
& =\frac{2 c x^{2}\left(n+2 c r^{2}+4 c r+c\right)+2 x\left(n+2 c r^{2}+5 c r+2 c\right)+r^{2}+3 r+2}{[n-c(r+1)][n-c(r+2)]} .
\end{aligned}
$$

Further, we have the recurrence relation

$$
\begin{aligned}
{[n-c(m+r+1)] V_{r, n, m+1}(x)=} & \phi^{2}(x)\left[V_{r, n, m}^{(1)}(x)+2 m V_{r, n, m-1}(x)\right] \\
& +[(1+2 c x)(m+r+1)-c x] V_{r, n, m}(x) .
\end{aligned}
$$

By using (2.1) and (2.2) the proof of the above lemma easily follows along the lines of $[6]$ and $[1]$.

It may be remarked that for all $x \in[0, \infty)$ (cases (i) and (ii)) and for all $x \in[0,1]$ (case (iii)), we have

$$
V_{r, n, m}(x)=O\left(n^{-[(m+1) / 2]}\right) .
$$

Lemma 2. If $f \in L_{p}^{r}[0, \infty) \cup \mathfrak{L}_{1}^{r}[0, \infty), 1<p \leq \infty$ and $x \in[0, \infty)$, we have

$$
M_{n}^{(r)}(f, x)=\alpha(n, r, c) \sum_{k=0}^{\infty} p_{n+c r, k}(x) \int_{0}^{\infty} b_{n-c r, k+r}(t) f^{(r)}(t) d t
$$

where

$$
\alpha(n, r, c)=\prod_{l=0}^{r-1} \frac{n+c l}{n-c(l+1)} .
$$

We see that the operators defined by (2.3) are not positive. To make the operators positive, we introduce the operators

$$
M_{n, r} f \equiv D^{r} M_{n} I^{r} f, \quad f \in L_{p}[0, \infty) \cup \mathfrak{L}_{1}[0, \infty),
$$

where $D$ and $I$ are the differential and integral operators respectively.

Therefore, we define the operators by

$$
\begin{aligned}
& M_{n, r}(f, x) \equiv \alpha(n, r, c) \sum_{k=0}^{\infty} p_{n+c r, k}(x) \int_{0}^{\infty} b_{n-c r, k+r}(t) f(t) d t, \\
& \quad f \in L_{p}[0, \infty) \cup \mathfrak{L}_{1}[0, \infty), n>(c+m) r .
\end{aligned}
$$


The operators $M_{n, r}$ are positive and the quantity $\left\|M_{n}^{(r)} f-f^{(r)}\right\|_{p}, f \in$ $L_{p}^{r}[0, \infty)$, is equivalent to $\left\|M_{n, r} f-f\right\|_{p}, f \in L_{p}[0, \infty)$.

Using (2.1), we can easily prove that for $n>c(r+1),\left\|M_{n, r} f\right\|_{1} \leq C\|f\|_{1}$ for $f \in L_{1}[0, \infty)$ and $\left\|M_{n, r} f\right\|_{\infty} \leq C\|f\|_{\infty}$ for $f \in L_{\infty}[0, \infty)$. Making use of the Riesz-Thorin theorem, we get

$$
\left\|M_{n, r} f\right\|_{p} \leq C\|f\|_{p}, \quad f \in L_{p}[0, \infty), 1 \leq p \leq \infty, n>c(r+1) .
$$

Corollary 3. For every $m \in \mathbb{N}^{0}, n>c(r+2 m+1)$ and $x \in[0, \infty)$, we have

$$
\begin{aligned}
\left|M_{n, r}\left((t-x)^{2 m}, x\right)\right| & \leq C n^{-m}\left(\phi^{2}(x)+n^{-1}\right)^{m}, \\
\left|M_{n, r}\left((t-x)^{2 m+1}, x\right)\right| & \leq C(1+2 x) n^{-m-1}\left(\phi^{2}(x)+n^{-1}\right)^{m},
\end{aligned}
$$

where the constant $C$ is independent of $n$. For fixed $x \in[0, \infty)$, we obtain

$$
\left|M_{n, r}\left((t-x)^{m}, x\right)\right|=O\left(n^{-[(m+1) / 2]}\right), \quad n \rightarrow \infty .
$$

Proof. Since $M_{n, r}\left((t-x)^{m}, x\right)=\alpha(n, r, c) V_{r, n, m}(x)$ the estimate $(2.5)$ follow from (2.2) along the lines of [6]; (2.6) immediately follows from (2.5).

Lemma 4. Let $t \in[0, \infty)$ and $n>c(r+m)$. Then

$$
M_{n, r}\left((1+t)^{-m}, x\right) \leq C(1+c x)^{-m}, \quad x \in[0, \infty),
$$

where the constant $C$ is independent of $n$.

Proof. It is easily verified that

$$
(1+c t)^{-m} b_{n-c r, k+r}(t)=\prod_{l=0}^{m-1} \frac{n-c r+l c}{n+l c+k c+1} b_{n-c r+m c, k+r}(t)
$$

and

$$
p_{n+c r, k}(x)=(1+c x)^{-m} \prod_{l=0}^{m} \frac{n+c r-l c+k c}{n+c r-l c} p_{n+c r-m c, k}(x) .
$$

Making use of these two identities and (2.1), we get

$$
\begin{aligned}
& M_{n, r}\left((1+t)^{-m}, x\right) \\
& \quad=\alpha(n, r, c) \sum_{k=0}^{\infty} p_{n+c r, k}(x) \int_{0}^{\infty} b_{n-c r, k+r}(t)(1+t)^{-m} d t \\
& \quad=\alpha(n, r, c) \sum_{k=0}^{\infty} p_{n+c r, k}(x) \prod_{l=0}^{m-1} \frac{n-c r+l c}{n+l c+k c+1} \int_{0}^{\infty} b_{n-c r+m c, k+r}(t) d t
\end{aligned}
$$




$$
\begin{aligned}
= & \alpha(n, r, c) \sum_{k=0}^{\infty}(1+c x)^{-m} p_{n+c r-m c, k}(x) \\
& \times \prod_{l=1}^{m} \frac{n+c r-l c+k c}{n+c r-l c} \prod_{l=0}^{m-1} \frac{n-c r+l c}{n+l c+k c+1} \\
\leq & C(1+c x)^{-m} \sum_{k=0}^{\infty} p_{n+c r-m c, k}(x)=C(1+c x)^{-m} .
\end{aligned}
$$

For the two monomials $e_{0}, e_{1}$ and $x \in[0, \infty), n \rightarrow \infty$, we obtain by direct computation

$$
\begin{gathered}
M_{n, r}\left(e_{0}, x\right)=1+O\left(n^{-1}\right), \\
M_{n, r}\left(e_{1}, x\right)=x\left(1+O\left(n^{-1}\right)\right) .
\end{gathered}
$$

Lemma 5. For

$$
H_{n}(u)=\left\{\int_{0}^{\infty} \int_{0}^{u}-\int_{0}^{u} \int_{0}^{\infty}\right\} \sum_{k=0}^{\infty} p_{n+c r, k}(x) b_{n-c r, k+r}(t)(u-t) d t d x,
$$

we have $H_{n}(u) \leq C n^{-1} \phi^{2}(u)$, where $C$ is independent of $n$ and $u$.

The proof easily follows by using (2.1) along the lines of [1, Lemma 5.2].

3. In this section, we prove the following global direct theorem.

Theorem 1. Suppose $f \in L_{p}[0, \infty), 1 \leq p<\infty, n>c(r+5)$. Then

$$
\left\|M_{n, r} f-f\right\|_{p} \leq C\left\{\omega_{\phi}^{2}\left(f, n^{-1 / 2}\right)+n^{-1}\|f\|_{p}\right\},
$$

where the constant $C$ is independent of $n$.

Pro of. By Taylor's expansion of $g$, we have

$$
g(t)=g(x)+(t-x) g^{\prime}(x)+\int_{x}^{t}(t-u) g^{\prime \prime}(u) d u
$$

Next, since $M_{n, r}(f, x)$ are uniformly bounded operators, for every $g \in$ $\bar{W}_{p}^{2}(\phi,[0, \infty))$, we have

$$
\left\|M_{n, r} f-f\right\|_{p} \leq C\|f-g\|_{p}+\left\|M_{n, r} g-g\right\|_{p} .
$$

Using (2.5), (2.8) and (3.1) and following [3], we have

$$
\begin{aligned}
\left\|M_{n, r} g-g\right\|_{p} \leq & C\left\{\|g\|_{p}+\left\|g^{\prime}\right\|_{L_{p}[0,1]}\right\} \\
& +\left\|(1+2 c r x) g^{\prime}\right\|_{L_{p}[0, \infty)}+\left\|M_{n, r}(R(g, t, x), \cdot)\right\|_{p} \\
\leq & C n^{-1}\left[\|g\|_{p}+\left\|\phi^{2} g^{\prime \prime}\right\|_{p}\right]+\left\|M_{n, r}(R(g, t, x), x)\right\|_{p}
\end{aligned}
$$

where $R(g, t, x)=\int_{x}^{t} g^{\prime \prime}(u)(t-u) d u$. 
Now, we prove that

$$
\left\|M_{n, r}(R(g, t, x), x)\right\|_{p} \leq C n^{-1}\left\|\left(\phi^{2}+n^{-1}\right) g^{\prime \prime}\right\|_{p} .
$$

We prove this for $p=1$ and $p=\infty$. The case $1<p<\infty$ follows again by the Riesz-Thorin theorem.

Using (2.5) for the case $m=1$ and Lemma 4 , the case $p=\infty$ easily follows (see e.g. [6]).

For $p=1$, we derive (3.4) by applying Fubini's theorem twice, the definition of $H_{n}(u)$ and Lemma 5:

$$
\begin{aligned}
\int_{0}^{\infty} \mid M_{n, r}( & R(g, t, x), x) \mid d x \\
\leq & \alpha(n, r, c) \int_{0}^{\infty} \sum_{k=0}^{\infty} p_{n+c r, k}(x) \int_{0}^{\infty} b_{n-c r, k+r}(t)\left|\int_{x}^{t} g^{\prime \prime}(u)(t-u) d u\right| d t d x \\
= & \alpha(n, r, c) \int_{0}^{\infty}\left|g^{\prime \prime}(u)\right|\left\{\int_{0}^{\infty} \int_{0}^{u}-\int_{0}^{u} \int_{0}^{\infty}\right\}(u-t) \\
& \times \sum_{k=0}^{\infty} p_{n+c r, k}(x) b_{n-c r, k+r}(t) d t d x d u \\
= & \alpha(n, r, c) \int_{0}^{\infty}\left|g^{\prime \prime}(u)\right| H_{n}(u) d u \\
\leq & C n^{-1}\left\|\phi^{2} g^{\prime \prime}\right\|_{1} \leq C n^{-1}\left\|\left(\phi^{2}+n^{-1}\right) g^{\prime \prime}\right\|_{1},
\end{aligned}
$$

where $C$ is independent of $n$. Hence (3.4) holds by the Riesz-Thorin theorem for $1 \leq p<\infty$. Combining the estimates (3.2), (3.3) and (3.4), we get

$$
\begin{aligned}
\left\|M_{n, r} f-f\right\|_{p}= & C\|f-g\|_{p}+C n^{-1}\left\{\|f-g\|_{p}+\|f\|_{p}\right. \\
& \left.+\left\|\phi^{2} g^{\prime \prime}\right\|_{p}+\left\|\left(\phi^{2}+n^{-1}\right) g^{\prime \prime}\right\|_{p}\right\} \\
\leq & C\left\{\|f-g\|_{p}+n^{-1}\left\|\phi^{2} g^{\prime \prime}\right\|_{p}+n^{-2}\left\|g^{\prime \prime}\right\|_{p}+n^{-1}\|f\|_{p}\right\} .
\end{aligned}
$$

Next taking the infimum over all $g \in \bar{W}_{p}^{2}(\phi,[0, \infty))$ on the right hand side, we get

$$
\left\|M_{n, r} f-f\right\|_{p} \leq C\left\{\bar{K}_{\phi}^{2}\left(f, n^{-1}\right)+n^{-1}\|f\|_{p}\right\} .
$$

This completes the proof of Theorem 1 .

R e mark. The conclusion of the above theorem is true for the space $L_{p}[0, \infty), 1 \leq p<\infty$ (i.e. $\lim _{n \rightarrow \infty}\left\|M_{n, r} f-f\right\|_{p}=0$ for every $f \in$ $\left.L_{p}[0, \infty)\right)$ since the basic fact about the Ditzian-Totik modulus of smooth- 
ness $\omega_{\phi}^{2}\left(f, n^{-1}\right)$ is that

$$
\lim _{n \rightarrow \infty} \omega_{\phi}^{2}\left(f, n^{-1}\right)=0 \quad \text { for all } f \in L_{p}[0, \infty) \text { if } 1 \leq p<\infty,
$$

or for all bounded functions $f \in C[0, \infty)$ which satisfy

$$
\lim _{x \rightarrow \infty} f(x)=L_{\infty}<\infty
$$

if $p=\infty$ (cf. [3, p. 36]).

\section{References}

[1] M. M. Derriennic, Sur l'approximation de fonctions intégrables sur $[0,1]$ par des polynômes de Bernstein modifiés, J. Approx. Theory 31 (1981), 325-343.

[2] Z. Ditzian and K. Ivanov, Bernstein type operators and their derivatives, ibid. 56 (1989), 72-90.

[3] Z. Ditzian and V. Totik, Moduli of Smoothness, Springer Ser. Comput. Math. 9, Springer, Berlin, 1987.

[4] J. L. Durrmeyer, Une formule d'inversion de la transformée de Laplace: Applications à la théorie des moments, Thèse de 3e Cycle, Faculté des Sciences de l'Université de Paris, 1967.

[5] V. Gupta, A note on modified Baskakov type operators, Approx. Theory Appl. 10 (1994), 74-78.

[6] M. Heilmann, Direct and converse results for operators of Baskakov-Durrmeyer type, ibid. 5 (1989), 105-127.

[7] H. S. Kasana, P. N. Agrawal and V. Gupta, Inverse and saturation theorems for linear combination of modified Baskakov operators, ibid. 7 (1991), 65-82.

[8] S. M. Mazhar and V. Totik, Approximation by modified Szász operators, Acta Sci. Math. (Szeged) 49 (1985), 257-269.

[9] A. Sahai and G. Prasad, On simultaneous approximation by modified Lupas operators, J. Approx. Theory 45 (1985), 122-128.

[10] R. P. Sinha, P. N. Agrawal and V. Gupta, On simultaneous approximation by modified Baskakov operators, Bull. Soc. Math. Belg. Sér. B 42 (1991), 217-231.

Department of Mathematics

University of Roorkee

Roorkee 247 667, India

E-mail: maths\%rurkeu@sirnetd.ernet.in
Current address of Vijay Gupta: Department of Mathematics Institute of Engineering and Technology Rohilkhand University Bareilly 243006, India 